\title{
Socioeconomic disparities in colorectal cancer survival: contributions of prognostic factors in a large Australian cohort
}

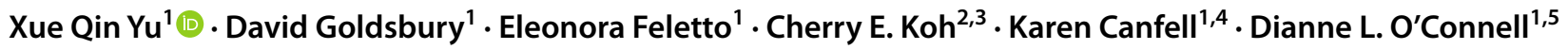

Received: 29 July 2021 / Accepted: 10 November 2021 / Published online: 25 November 2021

(c) The Author(s), under exclusive licence to Springer-Verlag GmbH Germany, part of Springer Nature 2021

\begin{abstract}
Purpose We quantified the contributions of prognostic factors to socioeconomic disparities in colorectal cancer survival in a large Australian cohort.

Methods The sample comprised 45 and Up Study participants (recruited 2006-2009) who were subsequently diagnosed with colorectal cancer. Both individual (education attained) and neighbourhood socioeconomic measures were used. Questionnaire responses were linked with cancer registrations (to December 2013), records for hospital inpatient stays, emergency department presentations, death information (to December 2015), and Medicare and Pharmaceutical Benefits claims for subsidised procedures and medicines. Proportions of socioeconomic survival differences explained by prognostic factors were quantified using multiple Cox proportional hazards regression.

Results 1720 eligible participants were diagnosed with colorectal cancer after recruitment: 1174 colon and 546 rectal cancers. Significant colon cancer survival differences were only observed for neighbourhood socioeconomic measure $(p=0.033)$ : $\mathrm{HR}=1.55 ; 95 \%$ CI 1.09-2.19 for lowest versus highest quartile, and disease-related factors explained $95 \%$ of this difference. For rectal cancer, patient- and disease-related factors were the main drivers of neighbourhood survival differences (28-36\%), while these factors and treatment-related factors explained $24-41 \%$ of individual socioeconomic differences. However, differences remained significant for rectal cancer after adjusting for all these factors.

Conclusion In this large contemporary Australian cohort, we identified several drivers of socioeconomic disparities in colorectal cancer survival. Understanding of the role these contributors play remains incomplete, but these findings suggest that improving access to optimal care may significantly reduce these survival disparities.
\end{abstract}

Keywords Colorectal cancer $\cdot$ Socioeconomic status $\cdot$ Survival disparity $\cdot$ Cancer epidemiology

\section{Introduction}

Xue Qin Yu

xueqiny@nswcc.org.au

1 The Daffodil Centre, The University of Sydney, a joint venture with Cancer Council New South Wales, Kings Cross, P O Box 572, Sydney, NSW 1340, Australia

2 Department of Colorectal Surgery, Royal Prince Alfred Hospital, Sydney, NSW, Australia

3 Discipline of Surgery, Central Clinical School, The University of Sydney, Sydney, NSW, Australia

4 Prince of Wales Clinical School, University of New South Wales, Sydney, NSW, Australia

5 School of Medicine and Public Health, University of Newcastle, Newcastle, NSW, Australia
Colorectal cancer (CRC) is the third-most common cancer and second-leading cause of cancer death worldwide (Bray et al. 2018). In many developed countries, including Australia, there have been substantial improvements in CRC survival in recent decades, due to advances in patients' care (Butler et al. 2013; Chawla et al. 2013). However, not all patients appear to have benefitted equally from these advances, with socioeconomic disparities in CRC survival being reported over the past decades (Beckmann et al. 2016; Stanbury et al. 2016a; Woods et al. 2006; Yu et al. 2008). The reasons for these survival differences are not well understood, although differences in disease stage at diagnosis, access and quality of treatment, and patients' characteristics are likely to be the potential causes (Woods et al. 2006). 
Identifying factors influencing CRC survival are crucial first steps towards addressing and reducing disparities in survival outcomes (Woods et al. 2006). In Australia, studies have attempted to disentangle the impact of several prognostic factors on socioeconomic disparities in CRC survival, and found that patients residing in higher socioeconomic neighbourhoods had longer survival after adjusting for stage at diagnosis, treatment received, and several other prognostic factors (Beckmann et al. 2016; Kelsall et al. 2009). However, these previous studies were limited by a relatively small sample size and lack of information on comorbidities (Kelsall et al. 2009), or not having individual socioeconomic measures or information on lifestyle factors (Beckmann et al. 2016), all of which may be associated with CRC patients' survival (Boyle et al. 2013; Woods et al. 2006). In this study, we investigated potential prognostic factors underlying survival disparities in CRC in a large Australian cohort (Banks et al. 2008), and quantified their contributions to the disparities.

\section{Methods}

The Sax Institute's 45 and Up Study is an ongoing Australian prospective cohort study of 267,153 people aged $\geq 45$ years residing in New South Wales (NSW), Australia during 2006-2009 (Banks et al. 2008). Prospective participants were randomly sampled from Services Australia (formerly the Australian Government Department of Human Services) enrolment database, which provides near complete coverage of the population. People aged $\geq 80$ years and residents of rural and remote areas were oversampled. Of those invited, $18 \%$ participated and participants included about $11 \%$ of the NSW population aged $\geq 45$ years. A detailed description of the analytical approaches used in this study has previously been published (Yu et al. 2019). Briefly, the study sample comprised participants of the 45 and Up Study who were diagnosed with colon (C18), or rectal cancer (C19-C20) during follow-up, identified by linkage to the populationbased NSW Cancer Registry up to December 2013.

To provide data on the patients' care pathway, and vital status, the survey data were linked with the NSW Admitted Patient Data Collection (APDC), NSW Emergency Department Data Collection (EDDC), Medicare Benefits Schedule (MBS) and Pharmaceutical Benefits Scheme (PBS) claims databases, and Cause of Death Unit Record File (COD-URF) (dates of availability of these health-related datasets are shown in the Supplementary Figure). Linkage to MBS and PBS records is facilitated by the Sax Institute using a unique identifier provided by Services Australia. All other datasets were probabilistically linked by the Centre for Health Record Linkage using a privacy-preserving approach to linkage (Centre for Health Record Linkage 2018).

\section{Exclusions}

Participants with any record of cancer before recruitment (either self-reported at baseline or those with a cancer diagnosis recorded in the NSW Cancer Registry between 1994 and baseline) were excluded, along with people first diagnosed at death or whose survival time was 0 days. Participants whose healthcare was subsidised by the Australian Government's Department of Veterans' Affairs were excluded as their prescription medicines have a separate billing arrangement and these data were not available. Those aged $\geq 85$ years at diagnosis were also excluded since misclassification of cause of death has been shown to be more common for older cancer patients (Dekker et al. 2014; Makkar et al. 2018).

\section{Outcome and exposure measures}

A patient's vital status was determined by linkage with COD-URF. Survival time was calculated from the date of diagnosis to the date of death from CRC or censored at the date of death from another cause or at the end of follow-up (December 2015).

Relative survival could not be estimated because relevant population life tables were not available, so we used the Surveillance, Epidemiology, and End Results causespecific death classification (Howlader et al. 2010) to optimise the accuracy of the survival estimates as causes of death recorded in population-based registries can be inaccurate (Yin et al. 2011).

To take advantage of the data available and adhere to previous recommendations (Chang et al. 2012; Wallner and Griggs 2018), two measures of socioeconomic status (SES) were used in this study. Individual-level SES was determined using the patient's highest level of education attainment self-reported at baseline and classified as 'low' (school certificate or below), 'medium' (higher school certificate, or trade/apprenticeship), or 'high' (certificate/diploma, or university degree). Neighbourhood SES (nSES) was based on where the participant lived at baseline and grouped into four categories using quartiles of the state-wide distribution of an index of relative socioeconomic disadvantage from the 2011 Australian Census (Australian Bureau of Statistics 2013). This index represents the average SES of people living within a given neighbourhood, including education, employment and occupation variables (Australian Bureau of Statistics 2013).

A joint SES variable was derived to examine any possible interaction of the two SES measures on patients' 
survival (Chang et al. 2012). nSES was split into low nSES (quartiles 1-2) and high nSES (quartiles 3-4), resulting in six categories: "high nSES/high education", "high nSES/ medium education", "high nSES/low education", "low nSES/high education", "low nSES/medium education", and "low nSES/low education".

\section{Prognostic factors}

The prognostic factors were grouped into four broad categories: patient-, lifestyle-, disease-, or treatment-related. Patient-related factors were obtained from the baseline questionnaire, apart from comorbidities, including marital status (married/de facto or other), private health insurance status (yes/no) (Banks et al. 2009), and place of residence at recruitment (major cities or non-major cities) using the Australian Standard Geographic Classification Remoteness Structure (Australian Bureau of Statistics 2003). Comorbidities were measured using the Charlson Comorbidity Index (Charlson et al. 1987) using hospitalisation records for five years before diagnosis $(0, \geq 1)$ (Yap et al. 2018).

Data on lifestyle factors were derived from the baseline questionnaire, including tobacco smoking (ever smoker, never smoker including those former smokers who quit $>15$ years ago because their risk of many comorbid conditions is close to that of never smokers (Moyer and Force 2014)), alcohol consumption (0,1-14, > 14 standard drinks per week), and physical activity classified as sedentary (0 min per week), insufficient (1-149 min), sufficient (150-299 min) or high (300+ minutes) (Yu et al. 2019). Body mass index (BMI) was calculated using self-reported height and weight $\left(<25,25-29.9, \geq 30 \mathrm{~kg} / \mathrm{m}^{2}\right)$ (National Health and Medical Research Council 2013).

Disease-related factors were derived from multiple data sources. Cancer stage at diagnosis from the Cancer Registry was grouped as localised, regional, distant, or unknown (Stanbury et al. 2016a). A recent assessment of this stage variable showed it to be an adequate surrogate staging system comparable to AJCC-TNM stage (Lawrance et al. 2019). Whether the patient's diagnosis followed an emergency presentation (yes/no) was determined by the date of diagnosis and dates of emergency department arrival and departure. As there is no uniform definition of emergency presentation before cancer diagnosis (Zhou et al. 2017), we used up to 14 days prior as the cut-point.

Anticancer treatments received were obtained from multiple sources and coded as yes/no based on any indication of treatment being provided according to the APDC, and MBS or PBS claims. Any such treatment (surgery, radiation therapy or systemic treatment) relating to CRC received up to six months after diagnosis was considered as a first course of treatment.

\section{Statistical analyses}

Patients with colon and rectal cancers were analysed separately after initial exploratory analysis suggested different survival patterns by education level $\left(P_{\text {interaction }}=0.022\right)$. Differences in prognostic factors between socioeconomic groups were tested using the ANOVA $F$ test for continuous variables and the $\chi^{2}$ test for categorical variables.

To better understand drivers of disparities in cancer outcomes like previous studies (Ellis et al. 2018; Hill et al. 2010; Ren et al. 2019; Seneviratne et al. 2015), we examined the proportions of the socioeconomic disparity in CRC survival explained separately by each group of prognostic factors using multiple Cox proportional hazards regression (Cox 1972). The hazard ratios (HRs) and 95\% confidence intervals (CIs) were estimated, with the highest SES category as the reference. Model one adjusted for sex, age, and year of diagnosis. Where significant differences in survival for SES were found in Model one, each group of prognostic factors (patient-, lifestyle-, disease-, and treatment-related factors) was then added to Model one individually. We first calculated overall disparity by subtracting one from the highest HR derived from Model one (e.g. the HR for nSES quartile 1 relative to nSES quartile 4) (Ren et al. 2019). The change in the highest HR for the same comparison after the addition of each group of factors was used to estimate the individual contribution of each group to the overall disparity. We added each group of prognostic factors individually, rather than adding them into Model one sequentially, because the order in which the factors are added could affect the estimate of the individual contribution of each (Seneviratne et al. 2015).

The final model included the variables in Model one plus all factors with $\mathrm{p}$ value $<0.15$ (Bursac et al. 2008), and stratified by stage to improve the model fit. All analyses were performed using SAS version 9.4. Tests for statistical significance were two-sided, $\alpha=0.05$.

\section{Results}

Of the 267,153 participants in the 45 and Up Study, 1720 eligible participants were diagnosed with CRC at age $<85$ years during follow-up (Fig. 1): 1174 colon and 546 rectal cancers.

The frequency distributions for selected variables by SES groups are presented in Tables 1 and 2 (colon and rectal cancer, respectively). Overall, patients with low SES for both measures tended to be older and have more comorbidities than their counterparts in the highest SES categories. As expected, fewer patients with lower SES 
Fig. 1 Study sample selection flow chart. NSWCR: NSW Cancer Registry; DVA: Department of Veterans' Affairs

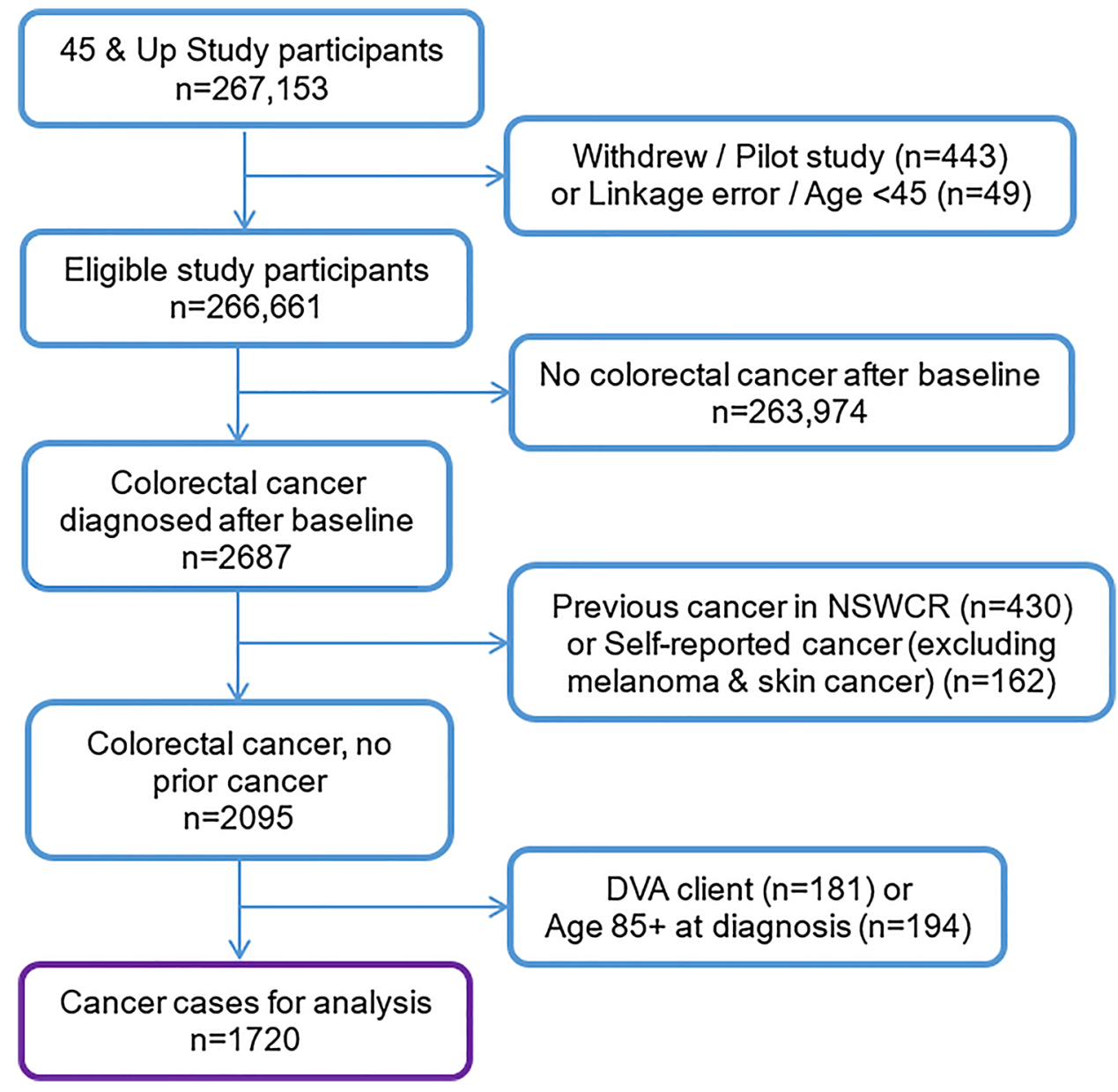

(both measures) had private health insurance coverage, compared to the highest SES categories for both colon and rectal cancers. Of note, there was no significant variation in the treatment received for colon and rectal cancers across nSES groups, whereas patients with low individual SES were less likely to receive surgery within six months for both colon and rectal cancers compared to those with high-level individual SES.

\section{Colon cancer survival}

Significant variation in colon cancer survival occurred only for $\mathrm{nSES}$, with HR = 1.55, 95\% CI 1.09-2.19 ( $p=0.033)$ for patients living in neighbourhoods in the lowest versus the highest quartile, and disease-related factors explained almost all the variation (Table 3). A similar pattern was observed for the joint SES measure where patients living in low nSES areas had a greater risk of colon cancer death regardless of their individual-level SES. In the final model, all factors (with $p<0.15$ ) together explained about $37 \%$ of the survival disparities, and variation in survival became non-significant $(p=0.12)$.

\section{Rectal cancer survival}

Greater variation was observed for rectal cancer survival, with a clear pattern of increasing hazards of death with lower SES for both SES measures (Table 4). With minimal adjustment (age, year of diagnosis and sex), the risk of dying from rectal cancer was tripled $(\mathrm{HR}=3.72,95 \% \mathrm{CI} 1.86-7.43)$ for patients living in neighbourhoods in the lowest, versus the highest quartile. After further adjustment, the disparities narrowed, with patient- and disease-related factors being the main contributors to the survival disparities. All the factors (with $p<0.15$ ) together in the final model explained $33 \%$ of the overall survival disparities by nSES, however, the survival disparities remained significant $(p=0.0044)$.

With only minimal adjustment, the estimated risk of dying for patients with rectal cancer who had low individuallevel SES was doubled $(\mathrm{HR}=2.26,95 \%$ CI 1.44-3.54) versus those who had high SES (Table 4). Further adjustment for patient-, disease- and treatment-related factors explained 33,41 , and $24 \%$ of the disparities, respectively. Survival disparities remained significant $(p=0.011)$, after adjusting for all the factors (with $p<0.15$ ) in the final model, which together explained $67 \%$ of the survival disparities. 
Table 1 Characteristics of colon cancer cases diagnosed during 2006-2013 among 45 and up study participants, NSW Australia

\begin{tabular}{|c|c|c|c|c|c|c|}
\hline & \multicolumn{6}{|c|}{ By neighbourhood socioeconomic measure } \\
\hline & Total & nSES 1 (Lowest) & nSES 2 & nSES 3 & nSES 4 (Highest) & $p$ value* \\
\hline No. of cases $(\%)$ & 1174 & $320(27 \%)$ & $331(28 \%)$ & $274(23 \%)$ & $249(21 \%)$ & \\
\hline \multicolumn{7}{|l|}{ Patients' characteristics } \\
\hline Age at diagnosis mean (SD), years & $70.0(8.9)$ & $71.5(8.6)$ & $69.4(8.8)$ & $69.6(9.4)$ & $69.5(8.7)$ & 0.0065 \\
\hline Male, $n(\%)$ & $585(49.8 \%)$ & $157(49.1 \%)$ & $154(46.5 \%)$ & $140(51.1 \%)$ & $134(53.8 \%)$ & 0.35 \\
\hline Marital status & & & & & & 0.0023 \\
\hline Married/de facto & $837(71.3 \%)$ & $205(64.1 \%)$ & $240(72.5 \%)$ & $197(71.9 \%)$ & $195(78.3 \%)$ & \\
\hline Private health insurance & & & & & & $<0.0001$ \\
\hline Yes & $699(59.5 \%)$ & $135(42.2 \%)$ & $186(56.2 \%)$ & $184(67.2 \%)$ & $194(77.9 \%)$ & \\
\hline Residence & & & & & & $<0.0001$ \\
\hline Major cities & $590(50.3 \%)$ & $117(36.6 \%)$ & $124(37.5 \%)$ & $151(55.1 \%)$ & $198(79.5 \%)$ & \\
\hline Non-Major cities & $584(49.7 \%)$ & $203(63.4 \%)$ & $207(62.5 \%)$ & $123(44.9 \%)$ & $51(20.5 \%)$ & \\
\hline Charlson comorbidity index & & & & & & 0.0005 \\
\hline 0 & $898(76.5 \%)$ & $220(68.8 \%)$ & $253(76.4 \%)$ & $226(82.5 \%)$ & $199(79.9 \%)$ & \\
\hline$\geq 1$ & $276(23.5 \%)$ & $100(31.2 \%)$ & $78(23.6 \%)$ & $48(17.5 \%)$ & $50(20.1 \%)$ & \\
\hline \multicolumn{7}{|l|}{ Lifestyle factors } \\
\hline Tobacco smoking & & & & & & 0.53 \\
\hline Ever smoker & $268(22.8 \%)$ & $69(21.6 \%)$ & $85(25.7 \%)$ & $61(22.3 \%)$ & $53(21.3 \%)$ & \\
\hline Never smoker ${ }^{\dagger}$ & $906(77.2 \%)$ & $251(78.4 \%)$ & $246(74.3 \%)$ & $213(77.7 \%)$ & $196(78.7 \%)$ & \\
\hline Alcoholic drinks (per week) & & & & & & $<0.0001$ \\
\hline 0 & $398(33.9 \%)$ & $136(42.5 \%)$ & $109(32.9 \%)$ & $99(36.1 \%)$ & $54(21.7 \%)$ & \\
\hline $1-14$ & $555(47.3 \%)$ & $138(43.1 \%)$ & $156(47.1 \%)$ & $125(45.6 \%)$ & $136(54.6 \%)$ & \\
\hline$>14$ & $209(17.8 \%)$ & $41(12.8 \%)$ & $60(18.1 \%)$ & $50(18.3 \%)$ & $58(23.3 \%)$ & \\
\hline Missing & $12(1.0 \%)$ & & & & & \\
\hline Moderate/vigorous physical activity & & & & & & 0.041 \\
\hline Sedentary & $68(5.8 \%)$ & $28(8.8 \%)$ & $18(5.4 \%)$ & $13(4.7 \%)$ & $9(3.6 \%)$ & \\
\hline Insufficient & $231(19.7 \%)$ & $72(22.5 \%)$ & $65(19.6 \%)$ & $52(19.0 \%)$ & $42(16.9 \%)$ & \\
\hline Sufficient & $152(12.9 \%)$ & $50(15.6 \%)$ & $35(10.6 \%)$ & $33(12.0 \%)$ & $34(13.7 \%)$ & \\
\hline High & $695(59.2 \%)$ & $164(51.3 \%)$ & $202(61.0 \%)$ & $168(61.3 \%)$ & $161(64.7 \%)$ & \\
\hline Unspecified & $28(2.4 \%)$ & & $11(3.3 \%)$ & $8(2.9 \%)$ & & \\
\hline Body mass index & & & & & & 0.17 \\
\hline Normal & $394(33.6 \%)$ & $96(30.0 \%)$ & $119(36.0 \%)$ & $87(31.8 \%)$ & $92(37.0 \%)$ & \\
\hline Overweight & $439(37.4 \%)$ & $114(35.6 \%)$ & $116(35.1 \%)$ & $115(42.0 \%)$ & $94(37.8 \%)$ & \\
\hline Obese & $270(23.0 \%)$ & $92(28.8 \%)$ & $76(23.0 \%)$ & $54(19.7 \%)$ & $48(19.3 \%)$ & \\
\hline Missing & $71(6.0 \%)$ & $18(5.6 \%)$ & $20(6.0 \%)$ & $18(6.6 \%)$ & $15(6.0 \%)$ & \\
\hline \multicolumn{7}{|l|}{ Disease-related factors } \\
\hline Cancer stage & & & & & & 0.062 \\
\hline Localised & $393(33.5 \%)$ & $99(30.9 \%)$ & $97(29.3 \%)$ & $103(37.6 \%)$ & $94(37.8 \%)$ & \\
\hline Regional & $479(40.8 \%)$ & $119(37.2 \%)$ & $148(44.7 \%)$ & $108(39.4 \%)$ & $104(41.8 \%)$ & \\
\hline Distant & $234(19.9 \%)$ & $78(24.4 \%)$ & $69(20.9)$ & $48(17.5 \%)$ & $39(15.7 \%)$ & \\
\hline Unknown & $68(5.8 \%)$ & $24(7.5 \%)$ & $17(5.1 \%)$ & $15(5.5 \%)$ & $12(4.8 \%)$ & \\
\hline \multicolumn{7}{|c|}{ Emergency presentation (prior to diagnosis) } \\
\hline 2-weeks prior & $115(9.8 \%)$ & $39(12.2 \%)$ & $33(10.0 \%)$ & $28(10.2 \%)$ & $15(6.0 \%)$ & 0.10 \\
\hline \multicolumn{7}{|l|}{ Treatment-related factors } \\
\hline Surgery in 6 months & $984(83.8 \%)$ & $262(81.9 \%)$ & $278(84.0 \%)$ & $236(86.1 \%)$ & $208(83.5 \%)$ & 0.57 \\
\hline \multirow[t]{3}{*}{ Systemic therapy in 6 months } & $428(36.5 \%)$ & $121(37.8 \%)$ & $127(38.4 \%)$ & $94(34.3 \%)$ & $86(34.5 \%)$ & 0.63 \\
\hline & \multicolumn{6}{|c|}{ By individual level SES } \\
\hline & Total & \multicolumn{2}{|l|}{ Low } & Medium & High & $p$ value* \\
\hline No. of cases & 1149 & \multicolumn{2}{|c|}{$491(43 \%)$} & $28(20 \%)$ & $430(37 \%)$ & \\
\hline
\end{tabular}


Table 1 (continued)

\begin{tabular}{|c|c|c|c|c|c|}
\hline & \multicolumn{5}{|c|}{ By individual level SES } \\
\hline & Total & Low & Medium & High & $p$ value* \\
\hline \multicolumn{6}{|l|}{ Patients' characteristics } \\
\hline Age at diagnosis mean (SD), years & $69.9(8.9)$ & $71.6(8.1)$ & $70.5(9.2)$ & $67.8(9.2)$ & $<0.0001$ \\
\hline Male, n (\%) & $574(50.0 \%)$ & $201(40.9 \%)$ & $155(68.0 \%)$ & $218(50.7 \%)$ & $<0.0001$ \\
\hline Marital status & & & & & 0.11 \\
\hline Married/de facto & $817(71.1 \%)$ & $333(67.8 \%)$ & $168(73.7 \%)$ & $316(74.5 \%)$ & \\
\hline Private health insurance & & & & & $<0.0001$ \\
\hline Yes & $687(59.8 \%)$ & $249(50.7 \%)$ & $123(54.0 \%)$ & $315(73.3 \%)$ & \\
\hline Residence & & & & & 0.096 \\
\hline Major cities & $578(50.3 \%)$ & $231(47.0 \%)$ & $114(50.0 \%)$ & $233(54.2 \%)$ & \\
\hline Non-Major cities & $571(49.7 \%)$ & $260(53.0 \%)$ & $114(50.0 \%)$ & $197(45.8 \%)$ & \\
\hline Charlson comorbidity index & & & & & $<0.0001$ \\
\hline 0 & $878(76.4 \%)$ & $350(71.3 \%)$ & $170(74.6 \%)$ & $358(83.3 \%)$ & \\
\hline$\geq 1$ & $271(23.6 \%)$ & $141(28.7 \%)$ & $58(25.4 \%)$ & $72(16.7 \%)$ & \\
\hline \multicolumn{6}{|l|}{ Lifestyle factors } \\
\hline Tobacco smoking & & & & & 0.91 \\
\hline Ever smoker & $262(22.8 \%)$ & $115(23.4 \%)$ & $51(22.4 \%)$ & $96(22.3 \%)$ & \\
\hline Never smoker ${ }^{\dagger}$ & $887(77.2 \%)$ & $376(76.6 \%)$ & $177(77.6 \%)$ & $334(77.7 \%)$ & \\
\hline Alcoholic drinks (per week) & & & & & $<0.0001$ \\
\hline 0 & $388(33.8 \%)$ & $219(44.6 \%)$ & $54(23.7 \%)$ & $115(26.7 \%)$ & \\
\hline $1-14$ & $545(47.4 \%)$ & $192(39.1 \%)$ & $113(49.6 \%)$ & $240(55.8 \%)$ & \\
\hline$>14$ & $207(18.0 \%)$ & $76(15.5 \%)$ & $57(25.0 \%)$ & $74(17.2 \%)$ & \\
\hline Missing & $9(0.8 \%)$ & & & & \\
\hline Moderate/vigorous physical activity & & & & & 0.0002 \\
\hline Sedentary & $67(5.8 \%)$ & $41(8.4 \%)$ & $14(6.1 \%)$ & $12(2.8 \%)$ & \\
\hline Insufficient & $223(19.4 \%)$ & $99(20.2 \%)$ & $53(23.3 \%)$ & $71(16.5 \%)$ & \\
\hline Sufficient & $150(13.1 \%)$ & $64(13.0 \%)$ & $28(12.3 \%)$ & $58(13.5 \%)$ & \\
\hline High & $683(59.4 \%)$ & $273(55.6 \%)$ & $124(54.4 \%)$ & $286(66.5 \%)$ & \\
\hline Unspecified & $26(2.3 \%)$ & & & & \\
\hline Body mass index & & & & & 0.075 \\
\hline Normal & $388(33.8 \%)$ & $158(32.2 \%)$ & $75(32.9 \%)$ & $155(36.1 \%)$ & \\
\hline Overweight & $432(37.6 \%)$ & $170(34.6 \%)$ & $97(42.5 \%)$ & $165(38.4 \%)$ & \\
\hline Obese & $263(22.9 \%)$ & $134(27.3 \%)$ & $42(18.4 \%)$ & $87(20.2 \%)$ & \\
\hline Missing & $66(5.7 \%)$ & $29(5.9 \%)$ & $14(6.1 \%)$ & $23(5.4 \%)$ & \\
\hline \multicolumn{6}{|l|}{ Disease-related factors } \\
\hline Cancer stage & & & & & 0.18 \\
\hline Localised & $385(33.5 \%)$ & $159(32.4 \%)$ & $81(35.5 \%)$ & $145(33.7 \%)$ & \\
\hline Regional & $471(41.0 \%)$ & $214(43.6 \%)$ & $76(33.3 \%)$ & $181(42.1 \%)$ & \\
\hline Distant & $227(19.8 \%)$ & $91(18.5)$ & $53(23.3 \%)$ & $83(19.3 \%)$ & \\
\hline Unknown & $66(5.7 \%)$ & $27(5.5 \%)$ & $18(7.9 \%)$ & $21(4.9 \%)$ & \\
\hline \multicolumn{6}{|c|}{ Emergency presentation (prior to diagnosis) } \\
\hline 2 weeks prior & $110(9.6 \%)$ & $58(11.8 \%)$ & $17(7.5 \%)$ & $35(8.1 \%)$ & 0.08 \\
\hline \multicolumn{6}{|l|}{ Treatment-related factors } \\
\hline Surgery in 6 months & $962(83.7 \%)$ & $405(82.5 \%)$ & $180(78.9 \%)$ & $377(87.7 \%)$ & 0.0096 \\
\hline Systemic therapy in 6 months & $421(36.6 \%)$ & $173(35.2 \%)$ & $80(35.1 \%)$ & $168(39.1 \%)$ & 0.42 \\
\hline
\end{tabular}

${ }^{*} p$ value for $F$ test for continuous variables and $\chi^{2}$ test for categorical variables

${ }^{\dagger}$ Never smoker includes those former smokers who quit $>15$ years ago

25 cases with missing value for education level 
Table 2 Characteristics of rectal cancer cases diagnosed during 2006-2013 among 45 and Up Study participants, NSW Australia

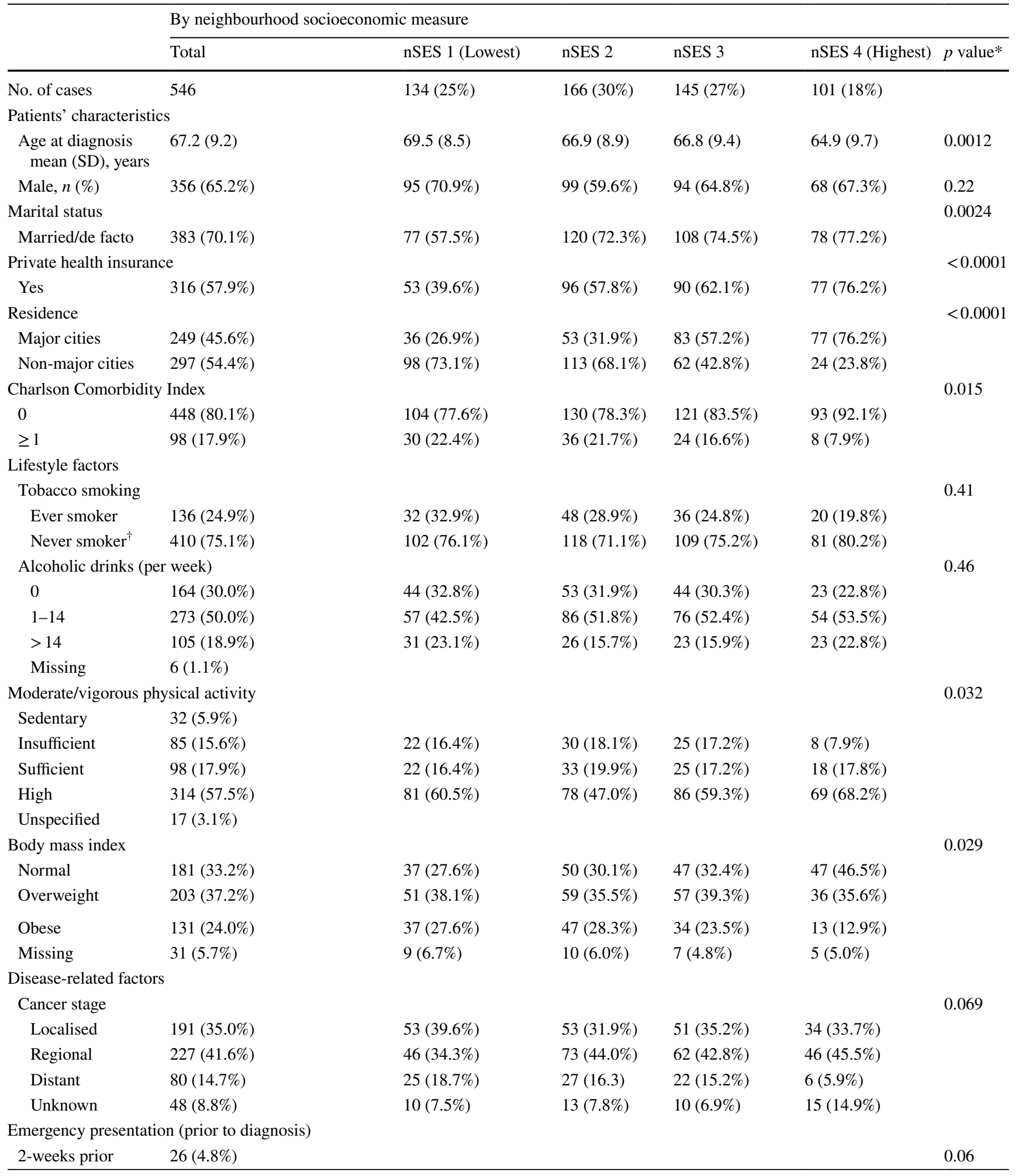


Table 2 (continued)

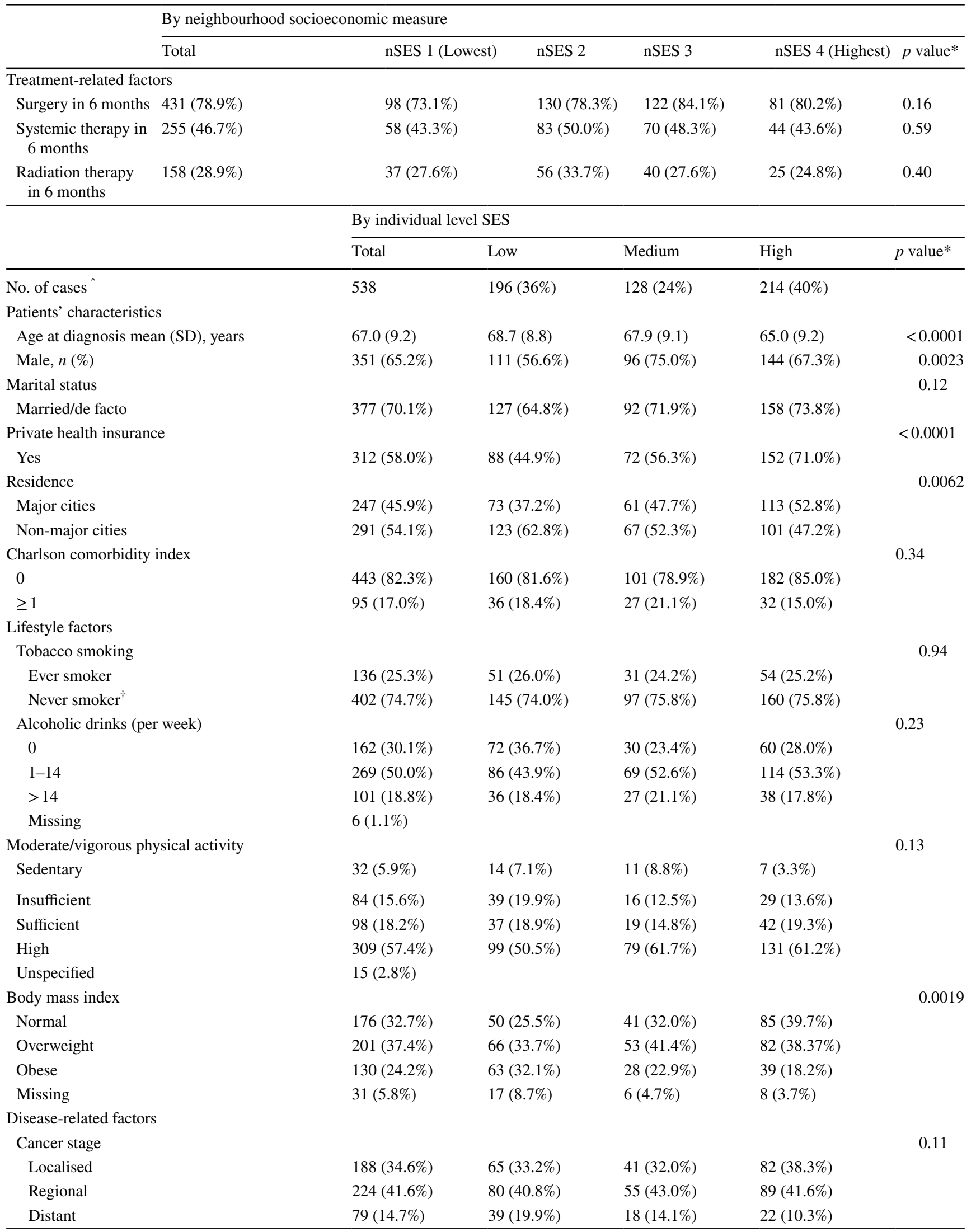


Table 2 (continued)

\begin{tabular}{|c|c|c|c|c|c|}
\hline & \multicolumn{5}{|c|}{ By individual level SES } \\
\hline & Total & Low & Medium & High & $p$ value* \\
\hline Unknown & $47(8.7 \%)$ & $12(6.1 \%)$ & $14(10.9 \%)$ & $21(9.8 \%)$ & \\
\hline \multicolumn{6}{|c|}{ Emergency presentation (prior to diagnosis) } \\
\hline 2-weeks prior & $26(4.8 \%)$ & & & $10(4.7 \%)$ & 0.46 \\
\hline \multicolumn{6}{|l|}{ Treatment-related factors } \\
\hline Surgery in 6 months & $425(79.0 \%)$ & $144(73.5 \%)$ & $97(75.8 \%)$ & $184(86.0 \%)$ & 0.0048 \\
\hline Systemic therapy in 6 months & $252(46.8 \%)$ & $83(42.4 \%)$ & $59(46.1 \%)$ & $110(51.4 \%)$ & 0.18 \\
\hline Radiation therapy in 6 months & $156(29.0 \%)$ & $48(24.5 \%)$ & $40(31.3 \%)$ & $68(31.8 \%)$ & 0.22 \\
\hline
\end{tabular}

${ }^{*} p$ value for $F$ test for continuous variables and $\chi^{2}$ test for categorical variables

Eight cases with missing values for individual-level SES

${ }^{\dagger}$ Never smoker includes those former smokers who quit $>15$ years ago

Regressions for the joint SES measure revealed similar patterns: those with low individual-level SES and living in a low nSES area had the highest risk of dying $(\mathrm{HR}=3.86,95 \%$ CI 2.02-7.37), versus those with high individual-level SES who lived in a high nSES area. Patient-, disease-, and treatment-related factors explained 35,38 and $21 \%$ of the overall variation, respectively. After including all the factors in the final model, $52 \%$ of the survival disparities were explained but the disparities remained significant $(p=0.0028)$.

Results for the final models for colon and rectal cancer can be found in Supplementary Tables 1-2.

\section{Discussion}

In this large Australian cohort study, we found that socioeconomic survival disparities continue to exist after a diagnosis of CRC, with the extent of this disparity varying by CRC sub-site and the SES measures used. By sub-site, the magnitude of the survival disparity was greater for rectal than for colon cancer. By SES measure, the magnitude of the survival disparity was greater for the neighbourhood measure than for an individual's SES. For the joint SES measure, we found that colon cancer patients living in a low SES neighbourhood had a higher risk of death, irrespective of their individual-level SES. Rectal cancer patients with high individual-level SES living in low nSES areas had over two times the risk of death compared to their counterparts living in high nSES areas. This is in the context of the Australian healthcare system that provides universal health care coverage to residents, with free access to essential care, and private health insurance covering some of the costs of specialist care and treatment in private facilities.

While disease-related factors are the main driver for the observed survival disparity for colon cancer, we found treatment-related factors are also independent significant contributors to the SES disparity for colon cancer (Supplementary Table 1). In addition, we found that lifestyle factors (smoking status, alcohol consumption and physical activity) also contribute significantly to the SES disparity for rectal cancer (Supplementary Table 2). These results highlight the importance of early detection and optimal treatment in reducing survival disparities for both colon and rectal cancer.

Our study showed that differences in stage at diagnosis and emergency department presentation explained most of the neighbourhood survival disparities for colon cancer. For rectal cancer, the effects of those prognostic factors had less impact on the neighbourhood survival differences than for individual-level SES, with approximately two-thirds and one-third of the survival disparities, respectively, remaining unexplained. Our results suggest that adjustment for lifestyle factors had minimal impact on the survival disparities. This could be due to the similar patterns of these lifestyle factors by socioeconomic groups in the study cohort.

The socioeconomic survival disparities were greater for rectal cancer than for colon cancer and may be partially explained by differences in treatment patterns. Currently, surgical resection with stage-appropriate neoadjuvant combined-modality therapy is the main treatment for rectal cancer. Both the quality of surgical treatment and timing of initiation of neoadjuvant/adjuvant therapies may vary between patients in different socioeconomic groups, thus affecting their survival. Generally, more technically challenging surgical procedures are often required when treating rectal cancer (Archampong et al. 2010). The unexplained residual disparities in rectal cancer survival could be because patients with lower SES are less likely to receive optimal treatment (Chawla et al. 2013; te Marvelde et al. 2019), as prior research showed that CRC patients had similar survival outcomes regardless of socio-demographic background when receiving guideline-driven treatment in clinical trials (Nur et al. 2008; Unger et al. 2018; Yoon et al. 2015). Additionally, rectal cancer patients with low SES may have 


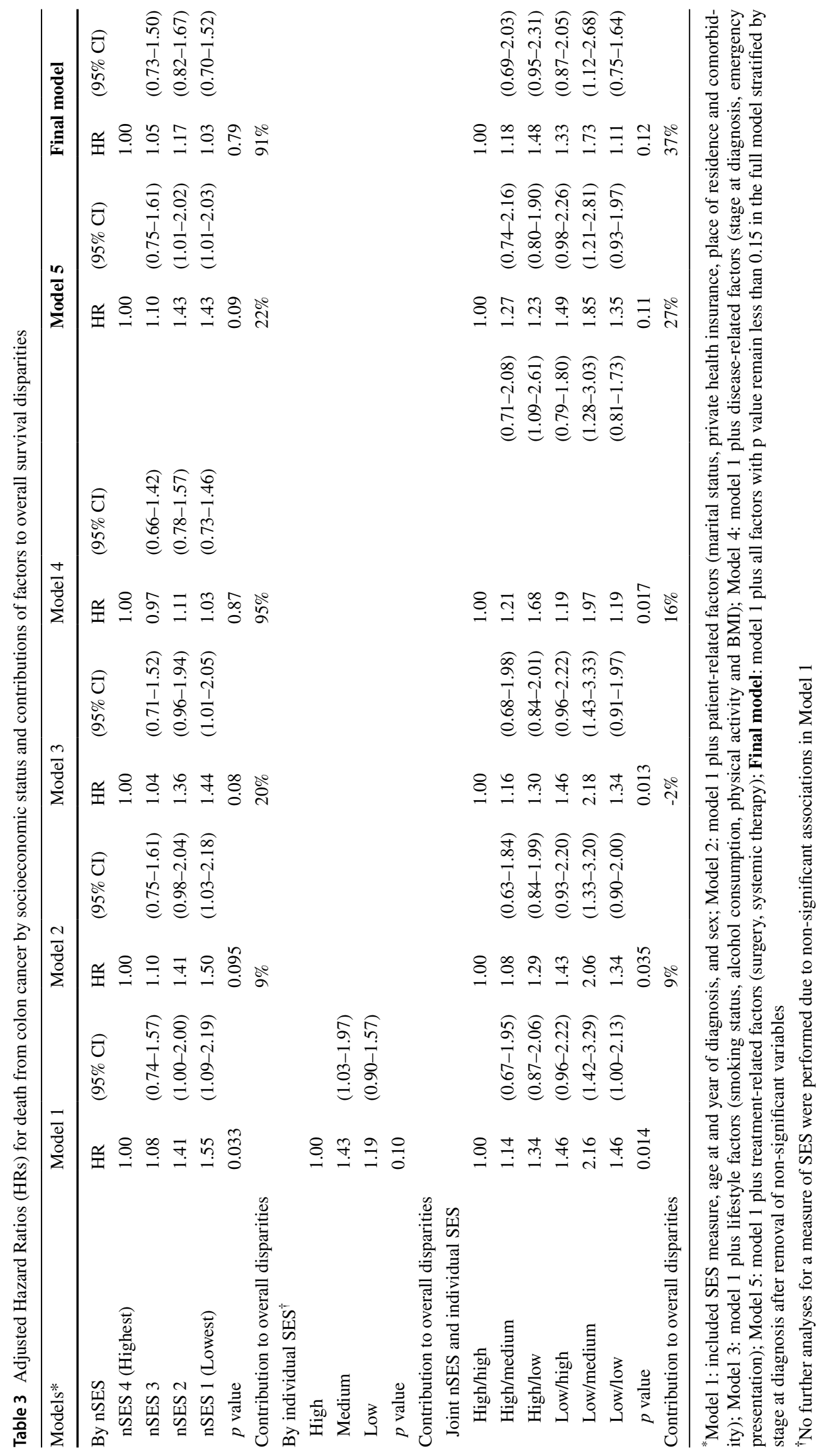




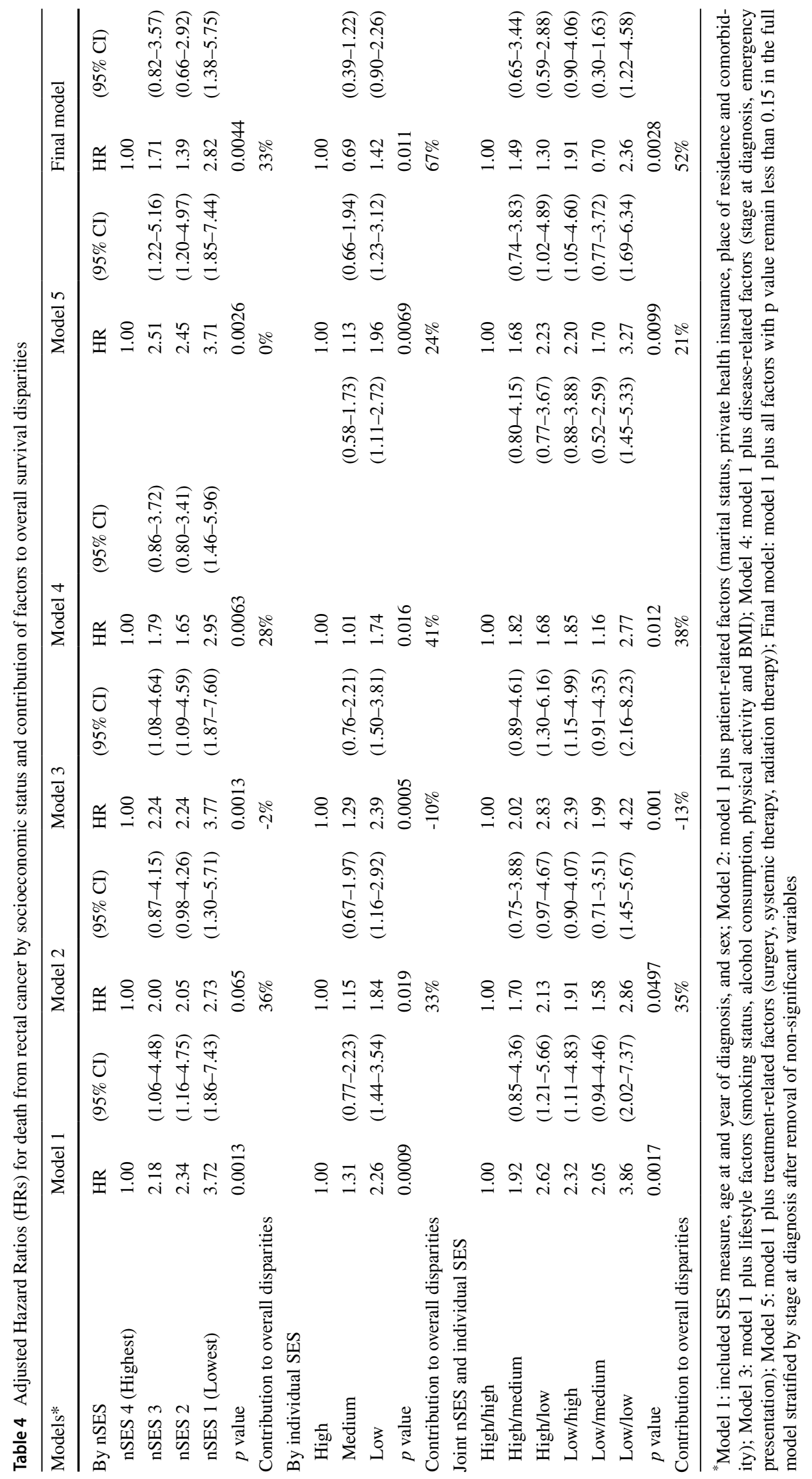


longer delay in getting adjuvant therapy after surgical resection due to lower adherence to effective therapy (Carethers and Doubeni 2020), and delaying adjuvant chemotherapy beyond 12 weeks post surgery was found to be associated with reduced survival for high-risk CRC patients (Biagi et al. 2011).

Our findings suggest that where people lived played a more important role than their education level in predicting CRC survival outcomes. The nSES variable used in this analysis is based on the smallest geographical unit for which a measure of SES from census data is available in Australia (Australian Bureau of Statistics 2013). It could be that nSES reflects access to cancer care and the quality of the care received (Ellis et al. 2018; Hill et al. 2010), and thus affects the outcomes of patients with both high and low education levels in the same neighbourhood. Timely and high-quality cancer care is a major prognostic factor, and this may be why our results indicate that nSES explains more of the survival disparities than individual patients' education level.

Survival disparities by SES may be partially attributable to the different screening patterns between population groups. Australia has a National Bowel Cancer Screening Program (NBCSP) which has been phased in since 2006 and reached full implementation in 2020 from which time all Australians aged 50-74 years are invited to screen biennially (Australian Institute of Health and Welfare 2019b). As of 2018, participation rates were lower for those living in the lowest socioeconomic areas (Australian Institute of Health and Welfare 2019b) which can often result in later stage at diagnosis. While our adjustment for cancer stage may account for some of the differences in screening participation, this adjustment was rather crude (Yu et al. 2005) and we are unable to control for possible within-stage survival differences for screen-detected versus symptomatic cancers (Lew et al. 2017). Nonetheless, as most of the participants in this study joined the cohort in 2008 and cancer diagnoses were up to 2013, the effect of screening on our survival results would be minimal.

This study has many strengths, such as the comprehensive inclusion of prospective data on patient and lifestyle variables from a large contemporary Australian cohort (Banks et al. 2008), and the ability to investigate a range of prognostic factors. Additionally, our analysis assessed these socioeconomic disparities at both individual and neighbourhood-levels separately and jointly, as has been previously recommended (Wallner and Griggs 2018). However, there are some potential limitations. First, the study population was limited to the participants in the 45 and Up Study, a cohort which has been shown to be older and more educated than the general population (Banks et al. 2008), so our results may not be representative of the entire NSW population. However prior studies have indicated that there is little evidence of bias in the association between nSES and cancer survival in this cohort (Creighton et al. 2018), and reasonable estimates of relative differences can be obtained (Mealing et al. 2010). Second, variables related to care coordination, or the quality of care were not available, each of which is potentially an important determinant of survival. Third, although individual's educational attainment is a widely used SES measure, using it alone has limitations, e.g., the older patients in our cohort were over-represented among those classified as less educated. Finally, coverage of radiation therapy may be incomplete as not all radiation therapy is captured in the MBS claims records for public patients in public hospitals (Australian Institute of Health and Welfare 2019a), and this information is limited in the hospital admission records (Goldsbury et al. 2012).

Our study was performed using data that pre-dates the COVID-19 pandemic. In Australia, as in other countries, one concern is that health service disruptions will have long term implications for cancer outcomes and that the pandemic will exacerbate pre-existing inequalities in outcomes. Although the NBCSP did not pause operations in Australia, indications of a drop in colonoscopy procedures during April-May 2020 were noted (Cancer Australia 2020). Our findings suggest that disparities in outcomes are in part driven by SES-related factors, particularly for rectal cancer. If SES-related factors prove to be important in determining cancer outcomes in the context of crisis-related health services disruptions, then addressing the impact of disparities in rectal cancer outcomes might be an important focus area for longer-term crisis mitigation planning (Carethers et al. 2020). Future policy decisions will need to consider ways in which disparities can be reduced and our work can help inform decisions that may relate to resource allocation or awareness program coverage, guided by the contribution of the various demographic and geographical factors.

As has been found previously (Beckmann et al. 2016; Kelsall et al. 2009; Stanbury et al. 2016a; b; Yu et al. 2005; Yu et al. 2008), our study showed that even in Australia, a country with a universal healthcare system, there are observed survival disparities by socioeconomic level. Our study goes beyond those previous studies by providing detailed analyses of contributing factors and the magnitude of their contribution. The approach used in this study to investigate underlying mechanisms for survival disparities could be a useful tool for improving our understanding of the underlying reasons for survival disparities. The approach can be applied to other cancer types both in Australia and in other countries. Using these findings, policy-driven and evidence-informed interventions could be developed to reduce SES-driven disparities and improve survival. These 
interventions could include encouraging lifestyle changes to reduce risky behaviours, improving participation in early detection programs, and ensuring delivery of high-quality treatments in concordance with treatment guidelines regardless of patient's socioeconomic level or where they live (Bergin et al. 2020; Carethers and Doubeni 2020; te Marvelde et al. 2019). The latter may require policy considerations regarding alternative funding models to support cancer patients with low SES. In addition, for rectal cancer further work is required to understand the remaining differences and how they can be addressed so that all rectal cancer patients have the best possible survival outcomes.

Acknowledgements This research was completed using data collected through the 45 and Up Study, managed by the Sax Institute (www.saxin stitute.org.au) in collaboration with their major partner Cancer Council NSW and partners: the Heart Foundation; NSW Ministry of Health; NSW Department of Communities and Justice; and Australian Red Cross Lifeblood. The Cause of Death Unit Record File (COD URF) is provided by the Australian Coordinating Registry for COD URF on behalf of Australian Registries of Births, Deaths and Marriages, Australian Coroners and the National Coronial Information System. We thank the many thousands of people participating in the 45 and Up Study, the Centre for Health Record Linkage for the record linkage and the NSW Ministry of Health, Cancer Institute NSW and Services Australia for the use of their data, and Clare Kahn for editorial assistance.

Author contributions XQY conceived the original research idea with input from DLO'C. XQY performed the data analysis and wrote the initial draft of this paper. DG, EF, CEK, KC and DLO'C revised the manuscript critically. DG made significant contributions to the description of data sources and variables to be used in the analysis. All authors approved the final version of this paper.

Funding This project has not received any specific funding.

Availability of data and material Data underlying this article may be shared on request to the corresponding author with approval from the NSW Population \& Health Services Research Ethics Committee.

Code availability Not applicable.

\section{Declarations}

Conflict of interest $\mathrm{KC}$ is co-principal investigator of an investigatorinitiated trial of cytology and primary HPV screening in Australia ("Compass"), which is conducted and funded by the VCS Foundation, a government-funded health promotion charity. VCS has received equipment and a funding contribution for the Compass trial from Roche Molecular Systems and Ventana USA. However neither KC, nor her institution on her behalf (Cancer Council NSW) has received direct or indirect funding from industry for Compass or any other project. No other conflict of interests is declared.

Ethics approval The 45 and Up Study was approved by the University of NSW Human Research Ethics Committee (HREC 05035/ HREC 10186). This analysis was covered by ethics approval from the NSW Population and Health Services Research Ethics Committee (HREC/14/CIPHS/54).

\section{References}

Archampong D, Borowski DW, Dickinson HO (2010) Impact of surgeon volume on outcomes of rectal cancer surgery: a systematic review and meta-analysis. Surgeon 8:341-352

Australian Bureau of Statistics (2003) ASGC remoteness classification: purpose and use. Commonwealth of Australia, Canberra

Australian Bureau of Statistics (2013) Information paper: an introduction to socio-economic indexes for areas (SEIFA), 2011. Australian Bureau of Statistics, Canberra

Australian Institute of Health and Welfare (2019a) Cancer in Australia 2019. Australian Institue of Health and Welfare, Canberra

Australian Institute of Health and Welfare (2019b) National Bowel Cancer Screening Program: monitoring report 2019. Australian Institue of Health and Welfare, Canberra

Banks E, Redman S, Jorm L, Armstrong B, Bauman A, Beard J, Beral V, Byles J, Corbett S, Cumming R, Harris M, Sitas F, Smith W, Taylor L, Wutzke S, Lujic S (2008) Cohort profile: the 45 and up study. Int J Epidemiol 37:941-947

Banks E, Jorm L, Lujic S, Rogers K (2009) Health, ageing and private health insurance: baseline results from the 45 and Up Study cohort. Aust N Z Health Policy 6:16

Beckmann KR, Bennett A, Young GP, Cole SR, Joshi R, Adams J, Singhal N, Karapetis C, Wattchow D, Roder D (2016) Sociodemographic disparities in survival from colorectal cancer in South Australia: a population-wide data linkage study. BMC Health Serv Res 16:24

Bergin RJ, Thomas RJS, Whitfield K, White V (2020) Concordance between Optimal Care Pathways and colorectal cancer care: Identifying opportunities to improve quality and reduce disparities. J Eval Clin Pract 26:918-926

Biagi JJ, Raphael MJ, Mackillop WJ, Kong W, King WD, Booth CM (2011) Association between time to initiation of adjuvant chemotherapy and survival in colorectal cancer: a systematic review and meta-analysis. JAMA 305:2335-2342

Boyle T, Fritschi L, Platell C, Heyworth J (2013) Lifestyle factors associated with survival after colorectal cancer diagnosis. Br J Cancer 109:814-822

Bray F, Ferlay J, Soerjomataram I, Siegel RL, Torre LA, Jemal A (2018) Global cancer statistics 2018: GLOBOCAN estimates of incidence and mortality worldwide for 36 cancers in 185 countries. CA Cancer J Clin 68:394-424

Bursac Z, Gauss CH, Williams DK, Hosmer DW (2008) Purposeful selection of variables in logistic regression. Source Code Biol Med 3:17

Butler EN, Chawla N, Lund J, Harlan LC, Warren JL, Yabroff KR (2013) Patterns of colorectal cancer care in the United States and Canada: a systematic review. J Natl Cancer Inst Monogr 2013:13-35

Cancer AUSTRALIA (2020) Review of the impact of COVID-19 on medical services and procedures in Australia utilising MBS data: Skin, breast and colorectal cancers, and telehealth services. Cancer Australia, Surry Hills

Carethers JM, Doubeni CA (2020) Causes of socioeconomic disparities in colorectal cancer and intervention framework and strategies. Gastroenterology 158:354-367

Carethers JM, Sengupta R, Blakey R, Ribas A, D’Souza G (2020) Disparities in Cancer Prevention in the COVID-19 Era. Cancer Prev Res (phila) 13:893-896

Centre for Health Record Linkage (2018) Sydney: NSW Ministry of Health. http://www.cherel.org.au/. Accessed 30 Apr 2018

Chang CM, Su YC, Lai NS, Huang KY, Chien SH, Chang YH, Lian WC, Hsu TW, Lee CC (2012) The combined effect of individual and neighborhood socioeconomic status on cancer survival rates. PLoS ONE 7:e44325 
Charlson ME, Pompei P, Ales KL, Mackenzie CR (1987) A new method of classifying prognostic comorbidity in longitudinal studies: development and validation. J Chronic Dis 40:373-383

Chawla N, Butler EN, Lund J, Warren JL, Harlan LC, Yabroff KR (2013) Patterns of colorectal cancer care in Europe, Australia, and New Zealand. J Natl Cancer Inst Monogr 2013:36-61

COX DR (1972) Regression models and life-tables. J R Stat Soc Ser B 34:187-220

Creighton N, Purdie S, Soeberg M, Walton R, Baker D, Young J (2018) Self-selection in a population-based cohort study: impact on health service use and survival for bowel and lung cancer assessed using data linkage. BMC Med Res Methodol 18:84

Dekker JW, Gooiker GA, Bastiaannet E, van den Broek CB, van der Geest LG, van de Velde CJ, Tollenaar RA, Liefers GJ (2014) Cause of death the first year after curative colorectal cancer surgery; a prolonged impact of the surgery in elderly colorectal cancer patients. Eur J Surg Oncol 40:1481-1487

Ellis L, Canchola AJ, Spiegel D, Ladabaum U, Haile R, Gomez SL (2018) Racial and ethnic disparities in cancer survival: the contribution of tumor, sociodemographic, institutional, and neighborhood characteristics. J Clin Oncol 36:25-33

Goldsbury DE, Armstrong K, Simonella L, Armstrong BK, O'Connell DL (2012) Using administrative health data to describe colorectal and lung cancer care in New South Wales, Australia: a validation study. BMC Health Serv Res 12:387

Hill S, Sarfati D, Blakely T, Robson B, Purdie G, Chen J, Dennett E, Cormack D, Cunningham R, Dew K, McCreanor T, Kawachi I (2010) Survival disparities in Indigenous and non-Indigenous New Zealanders with colon cancer: the role of patient comorbidity, treatment and health service factors. J Epidemiol Community Health 64:117-123

Howlader N, Ries LA, Mariotto AB, Reichman ME, Ruhl J, Cronin KA (2010) Improved estimates of cancer-specific survival rates from population-based data. J Natl Cancer Inst 102:1584-1598

Kelsall HL, Baglietto L, Muller D, Haydon AM, English DR, Giles GG (2009) The effect of socioeconomic status on survival from colorectal cancer in the Melbourne Collaborative Cohort Study. Soc Sci Med 68:290-297

Lawrance S, Bui C, Mahindra V, Arcorace M, Cooke-Yarborough C (2019) Assessing a modified-AJCC TNM staging system in the New South Wales Cancer Registry. Aust BMC Cancer 19:850

Lew JB, St John DJB, Xu XM, Greuter MJE, Caruana M, Cenin DR, He EE, Saville M, Grogan P, Coupe VMH, Canfell K (2017) Long-term evaluation of benefits, harms, and cost-effectiveness of the National Bowel Cancer Screening Program in Australia: a modelling study. Lancet Public Health 2:e331-e340

Makkar N, Ostrom QT, Kruchko C, Barnholtz-Sloan JS (2018) A comparison of relative survival and cause-specific survival methods to measure net survival in cancer populations. Cancer Med 7:4773-4780

Mealing NM, Banks E, Jorm LR, Steel DG, Clements MS, Rogers KD (2010) Investigation of relative risk estimates from studies of the same population with contrasting response rates and designs. BMC Med Res Methodol 10:26

Moyer VA, Force USPST (2014) Screening for lung cancer: U.S. Preventive Services Task Force recommendation statement. Ann Intern Med 160:330-338

National Health and Medical Research Council (2013) Clinical Practice Guidelines for the Management of Overweight and Obesity in Adults, Adolescents and Children in Australia. Melbourne: NHMRC

Nur U, Rachet B, Parmar MK, Sydes MR, Cooper N, Lepage C, Northover JM, James R, Coleman MP (2008) No socioeconomic inequalities in colorectal cancer survival within a randomised clinical trial. Br J Cancer 99:1923-1928
Ren JX, Gong Y, Ling H, Hu X, Shao ZM (2019) Racial/ethnic differences in the outcomes of patients with metastatic breast cancer: contributions of demographic, socioeconomic, tumor and metastatic characteristics. Breast Cancer Res Treat 173:225-237

Seneviratne S, Campbell I, Scott N, Shirley R, Peni T, Lawrenson R (2015) Ethnic differences in breast cancer survival in New Zealand: contributions of differences in screening, treatment, tumor biology, demographics and comorbidities. Cancer Causes Control 26:1813-1824

Stanbury JF, Baade PD, Yu Y, Yu XQ (2016a) Cancer survival in New South Wales, Australia: socioeconomic disparities remain despite overall improvements. BMC Cancer 16:48

Stanbury JF, Baade PD, Yu Y, Yu XQ (2016b) Impact of geographic area level on measuring socioeconomic disparities in cancer survival in New South Wales, Australia: a period analysis. Cancer Epidemiol 43:56-62

te Marvelde L, McNair P, Whitfield K, Autier P, Boyle P, Sullivan R, Thomas RJS (2019) Alignment with indices of a care pathway is associated with improved survival. EClinicalMedicine 15:42-50

Unger JM, Moseley A, Symington B, Chavez-Macgregor M, Ramsey SD, Hershman DL (2018) Geographic distribution and survival outcomes for rural patients with cancer treated in clinical trials. JAMA Netw Open 1:e181235

Wallner LP, Griggs JJ (2018) Advancing the science of cancer health disparities research. J Clin Oncol 36:1-3

Woods LM, Rachet B, Coleman MP (2006) Origins of socio-economic inequalities in cancer survival: a review. Ann Oncol 17:5-19

Yap S, Goldsbury D, Yap ML, Yuill S, Rankin N, Weber M, Canfell K, O'Connell DL (2018) Patterns of care and emergency presentations for people with non-small cell lung cancer in New South Wales, Australia: a population-based study. Lung Cancer 122:171-179

Yin D, Morris CR, Bates JH, German RR (2011) Effect of misclassified underlying cause of death on survival estimates of colon and rectal cancer. J Natl Cancer Inst 103:1130-1133

Yoon HH, Shi Q, Alberts SR, Goldberg RM, Thibodeau SN, Sargent DJ, Sinicrope FA, Alliance for clinical trials in oncology (2015) Racial differences in BRAF/KRAS mutation rates and survival in stage III colon cancer patients. J Natl Cancer Inst. https://doi. org/10.1093/jnci/djv186

Yu XQ, O'Connell DL, Gibberd RW, Armstrong BK (2008) Assessing the impact of socio-economic status on cancer survival in New South Wales, Australia 1996-2001. Cancer Causes Control 19:1383-1390

Yu XQ, Goldsbury D, Yap S, Yap ML, O’Connell DL (2019) Contributions of prognostic factors to socioeconomic disparities in cancer survival: protocol for analysis of a cohort with linked data. BMJ Open 9:e030248

YU, X. Q., O'CONNELL D, L., GIBBERD, R. W. \& ARMSTRONG, B. K. (2005) A population-based study from New South Wales, Australia 1996-2001: area variation in survival from colorectal cancer. Eur J Cancer 41:2715-2721

Zhou Y, Abel GA, Hamilton W, Pritchard-Jones K, Gross CP, Walter FM, Renzi C, Johnson S, McPhail S, Elliss-Brookes L, Lyratzopoulos G (2017) Diagnosis of cancer as an emergency: a critical review of current evidence. Nat Rev Clin Oncol 14:45-56

Publisher's Note Springer Nature remains neutral with regard to jurisdictional claims in published maps and institutional affiliations. 